\title{
2.5 Gb/s Simple Optical Wireless Communication System for Particle Detectors in High Energy Physics
}

\author{
Wajahat Ali ${ }^{* \dagger}$ \\ Scuola Superiore Sant'Anna \\ E-mail: w.aliesssup.it

\section{Giulio Cossu} \\ Scuola superiore Sant'Anna \\ E-mail: giulio.cossulsssup.it

\section{Raffele Corsini} \\ Scuola superiore Sant'Anna \\ E-mail: r.corsiniesssup.it
}

\section{Ernesto Ciaramella}

Scuola superiore Sant'Anna

E-mail: e.ciaramelladsssup.it

\section{Roberto Dell'Orso}

INFN Pisa

E-mail: roberto.dellorso@pi.infn.it

\section{Alberto Messineo \\ INFN Pisa \\ E-mail: alberto.messineo@pi.infn.it}

\section{Fabrizio Palla}

INFN Pisa

E-mail: Fabrizio.Palla@cern.ch

\begin{abstract}
We demonstrated low cost and power-efficient a $2.5 \mathrm{~Gb} / \mathrm{s}$ optical wireless transmission system at $10 \mathrm{~cm}$ distance, which should be employed in high-energy physics experiments such as Compact Muon Solenoid. The system uses an off-the-shelf VCSEL and a PIN photodiode with proper ball lens. Its most attractive feature is that it does not need a complex active tracking system because its measured tolerance to misalignment is around $\pm 1 \mathrm{~mm}$ at Bit Error Rate of $10^{-12}$. The experimental results show that this can be a viable solution for future HEP experiments.
\end{abstract}

The 25th International workshop on vertex detectors

September 26-30, 2016

La Biodola, Isola d'Elba ITALY

*Speaker.

${ }^{\dagger}$ Supported by EU FP7-PEOPLE-2012-ITN project nr 317446, INFIERI 


\section{Introduction}

Compact Muon Solenoid (CMS) is one of the major experiment operating in the Large Hadron Collider (LHC) at CERN. The inner tracker of CMS is composed of approximately 10,000 silicon detector modules arranged in layers. The detector modules are fixed on concentric barrels which are separated by tens of centimeter [1]. In the current version of the tracker, approximately 40,000 optical fiber links are used for the readout of physics events generated by the silicon detector modules [2]. This increases the material budget, limits the space and also introduces excessive labor cost for cables installation and management. The large amount of non-sensitive material of the tracker also reduces the detector performance because of multiple scattering. In addition, future upgrades of the particle detectors will generate more data and thus higher data rate will be required. Any possibility to route new cables can be difficult due to space limitations.

High speed Optical Wireless Communication (OWC) is a promising solution because it can provide the radial connectivity between the layers of silicon detector modules reducing the complexity of an optical fiber networks. The data will be transmitted from the inner layers of detectors to the outer layers, which will be connected by optical fiber links as shown in Fig. 1. This technology will save numerous fiber links and will help in reduction of both complexity and material budget besides providing high data rate connectivity.

Only preliminary work has been performed to use the OWC system technology in HEP experiments. We will summarize briefly the proof of concept developed in the past. The initial study of OWC for HEP particle detectors was carried out by J. Charamowickz et al. in [3]. They demonstrated free-space transmission at data rates of $4 \mathrm{~Gb} / \mathrm{s}$ to $10 \mathrm{~Gb} / \mathrm{s}$ for $21 \mathrm{~cm}$ and $11 \mathrm{~cm}$ transmission distance respectively through $2 \mathrm{~mm}$ silicon samples. Because of the proof-of-concept nature of the paper, only few experimental details were provided by the authors and no analysis were performed to prove that the proposed setup could effectively work in HEP environments. Many design constraints have to be investigated e.g. proper selection of transmitter and receiver, mechanical tolerance to misalignment, beam divergence and its effect on data rates.

In another work, the Argonne National Lab [4] presented a conceptual OWC design with the transceiver based on a Mach-Zehnder Modulators (MZM), where the laser source would be placed outside the irradiation zone. The tolerance was provided by Micro Electro-Mechanical Systems (MEMS) devices for light beam steering. Although they provided good misalignment tolerance to the system, yet they add lots of complexity because of the control electronics (based on FPGA and DAC/ADC) needed to monitor the feedback, provided by secondary lasers and detectors. Besides the complexity, the authors did not investigate the radiation hardness of the devices (both optical and electronics). They may not work in harsh environments especially because of high radiation levels. The only device tested under irradiation is the MZM [5] but the research is still at its early stages, although showing promising results.

The mainstream research on laser-based OWC also used collimated beams with active alignment techniques [6, 7]. However, in the structure of particle detectors only passive alignment is possible because active tracking systems add complexity and material budget. Moreover, its control electronics may not be suitable for HEP environments.

To overcome these limitations, we designed and developed an OWC system that can work with a passive alignment with a higher tolerance as compared to the requirement of the CMS 


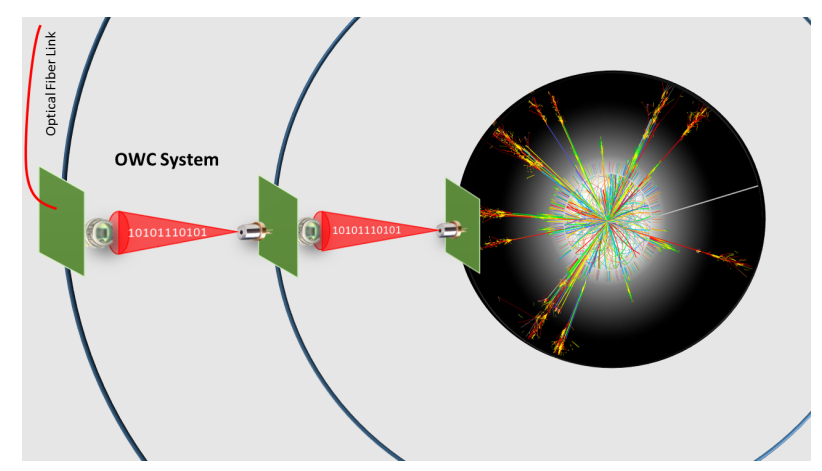

Figure 1: Conceptual diagram of OWC link in the inner tracker of CMS.

tracker (which is $\geq 0.25 \mathrm{~mm}$ ) [8]. In the preliminary work, we performed a ray-tracing simulation of a $2.5 \mathrm{~Gb} / \mathrm{s}$ OWC system based on VCSEL and PIN photodiode at $10 \mathrm{~cm}$ distance. From these simulations, the observed misalignment tolerance was greater than $\pm 0.8 \mathrm{~mm}[8]$. In this paper, we experimentally demonstrated the $2.5 \mathrm{~Gb} / \mathrm{s}$ OWC system using a single off-the-shelf VCSEL as the transmitter and a PIN photodiode with a ball lens as the receiver. The achieved transmission distance was $10 \mathrm{~cm}$ with a tolerance to misalignment of $\pm 1 \mathrm{~mm}$ provided by a proper choice of the receiver ball-lens. By this analysis, we realized a low-cost and energy efficient system prototype.

\section{2. $2.5 \mathrm{~Gb} / \mathrm{s}$ Experiment and results}

Based on the ray-tracing simulation made by the authors [8], we realized the experimental setup shown in Fig. 2. As we discussed, we considered a VCSEL because of its low cost and its low divergence angle with circular beam waist as compared to edge emitting lasers. In addition, VCSELs can be radiation resistant, which make them attractive for HEP applications [9].

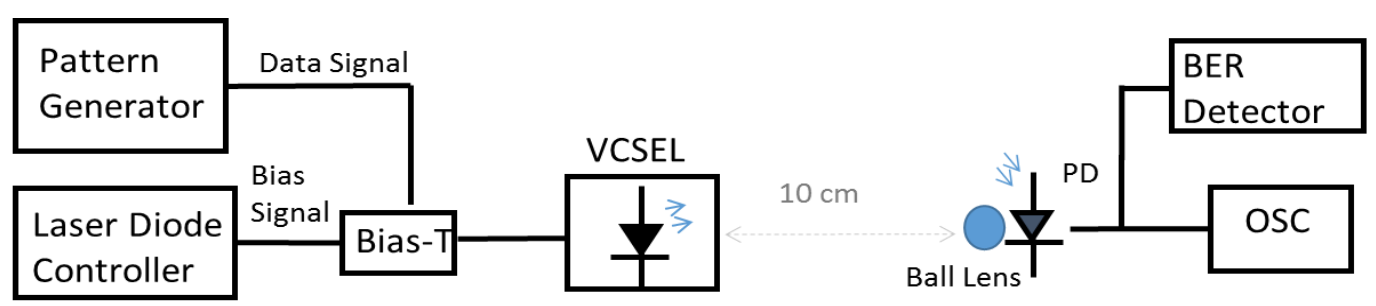

Figure 2: Experimental setup for optimizing photodiode with suitable ball lens at $2.5 \mathrm{~Gb} / \mathrm{s}$. OSC: Sampling oscilloscope; PD: PIN photodiode+TIA

The VCSEL (1550 nm, $1 \mathrm{~mW}$ optical output power and $16^{\circ}$ Full Width at Half Maximum divergence angle) was directly modulated at $2.5 \mathrm{~Gb} / \mathrm{s}$ by Pseudo Random Bit Sequence (PRBS) NRZ data with a length of $2^{15}-1$ bits. In order to simplify the tolerance measurements, the VCSEL was placed on a 3-axis translator stage. The receiver consisted of a PIN photodiode (sensitive area of $60 \mu \mathrm{m}, 3-\mathrm{dB}$ bandwidth of $1.8 \mathrm{GHz}$ ) with trans-impedance amplifier (TIA). Also, the photodiode was demonstrated to be radiation hard [9]. It was placed on a similar 3-axis translator stage to set the photodiode active area at the focal point of the ball lens. The transmission distance was fixed at $10 \mathrm{~cm}$ as required by the specification [1]. A ball lens was placed in front of the photodiode 


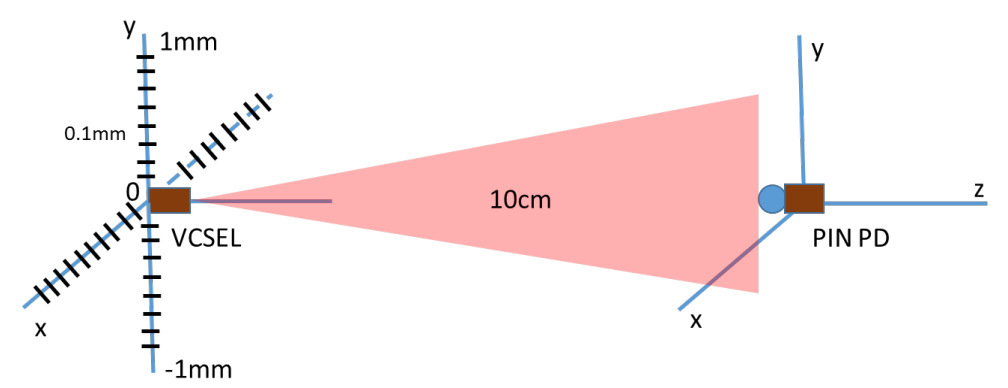

Figure 3: Misalignment tolerance measurement by moving VCSEL with $0.1 \mathrm{~mm}$ step on radial (x-y) axis

to increase the collected light. We selected fused silica and quartz glass type lenses, since they can provide proper radiation hardness, i.e. much better than BK7 glass at $1550 \mathrm{~nm}$ [10]. The TIA outputs (direct and inverted) were connected to a Bit Error Ratio (BER) detector and a sampling oscilloscope for eye diagram analysis. Three ball lenses $(3 \mathrm{~mm}, 4 \mathrm{~mm}$ and $5 \mathrm{~mm}$ diameter) were characterized to select the best one in terms of signal quality, tolerance values, required transmitted power and suitability for packaging. The signal quality of the OWC link is expressed by the Qfactor and is given by:

$$
Q=\frac{\mu_{1}-\mu_{0}}{\sigma_{1}+\sigma_{0}}
$$

which is widely used in optical communications to describe the receiver performance under Gaussian noise approximation. In eq. $2.1, \mu_{1,0}$ is the mean value while $\sigma_{1,0}$ is the standard deviation of the Gaussian noise of ' 1 ' and ' 0 ' levels. BER of $10^{-12}$ was targeted according to the CMS experiment requirements.

We started the characterization with the $5 \mathrm{~mm}$ ball lens in order to obtain the higher optical power at the photodiode. Both photodiode and VCSEL were aligned by maximizing the eye opening. Bias current of the laser $(4.7 \mathrm{~mA})$ and peak-to-peak voltage from the pattern generator $(1.130 \mathrm{Vpp})$ were also optimized in order to maximize the Q-factor. Figure 4 depicts the wide open eye diagram of $2.5 \mathrm{~Gb} / \mathrm{s}$ for $4 \mathrm{~mm}$ ball lens at aligned position $(\mathrm{x}=\mathrm{y}=0)$. Then, we misaligned the VCSEL along the radial distance ( $\mathrm{X}$ or $\mathrm{Y}$ axis, in both directions) by $0.1 \mathrm{~mm}$ step as illustrated in Fig. 3 and noted the BER and the Q-factor values. This process of misalignment was repeated until we lost the line of sight (LOS) completely. Same procedure was performed for $3 \mathrm{~mm}$ and $4 \mathrm{~mm}$ ball lenses.

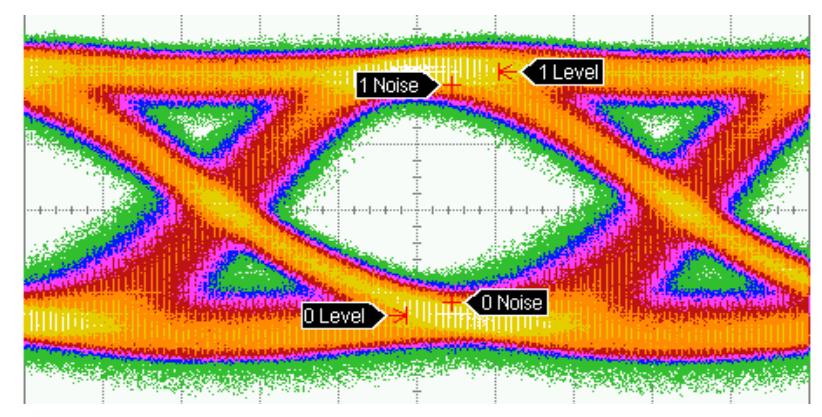

Figure 4: Eye diagram for $2.5 \mathrm{~Gb} / \mathrm{s}$ at $10 \mathrm{~cm}$ transmission. 

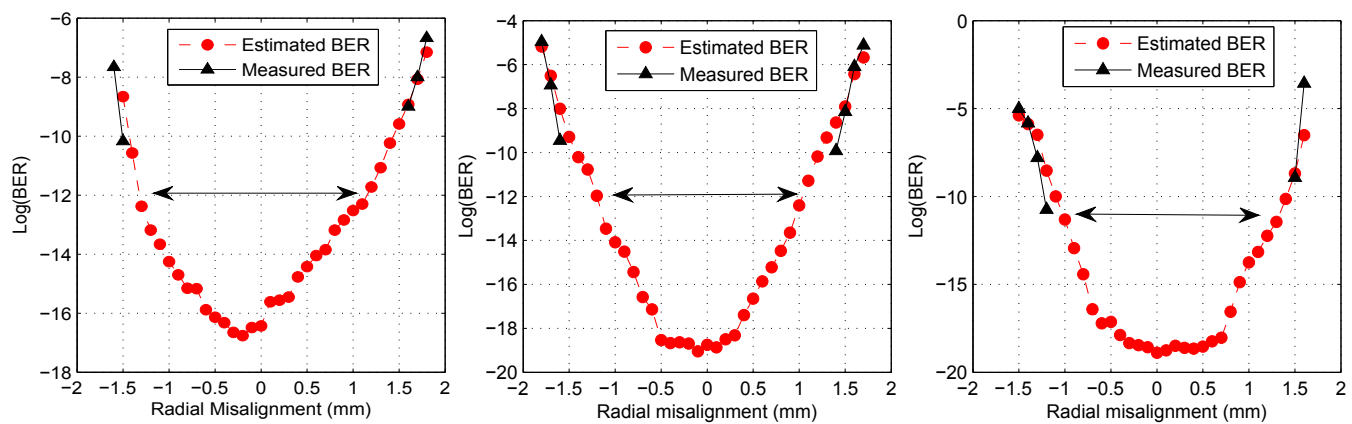

Figure 5: Tolerance to misalignment: BER vs radial displacement for $3 \mathrm{~mm}$ (a), $4 \mathrm{~mm}$ (b), $5 \mathrm{~mm}$ (c) ball lens

\begin{tabular}{|c|c|c|}
\hline & Tolerance @ BER 1e-12 & Maximum Q-factor \\
\hline $5 \mathrm{~mm}$ & $+/-1 \mathrm{~mm}$ & 8.95 \\
\hline $4 \mathrm{~mm}$ & $+/-1.1 \mathrm{~mm}$ & 8.95 \\
\hline $3 \mathrm{~mm}$ & $+/-1.24 \mathrm{~mm}$ & 8.53 \\
\hline
\end{tabular}

Figure 6: Summary table presenting maximum Q-factor values at aligned position and misalignment tolerance values of each ball lens at BER of $10^{-12}$.

Figures 5(a-c) report the BER values as a function of the radial misalignment and they show the alignment tolerance for the 3 lenses. The tolerance limit is defined as the misalignment range where the BER is $\leq 10^{-12}$. Because of the long measurement time ( $>30$ mins for $99 \%$ confidence level) for BER $\leq 10^{-12}$, we also estimated the BER values from the Q-factor exploiting the relationship between the two parameters, as given in [11]:

$$
B E R=\frac{1}{2} \operatorname{erfc}\left(\frac{Q}{\sqrt{2}}\right)
$$

As can be seen from Fig. 5(a-c), the estimated BER values were slightly worse than the measured values because of the non ideal Gaussian noise of the OWC system. We can observe that $4 \mathrm{~mm}$ and $3 \mathrm{~mm}$ ball lenses provided tolerance values of $\pm 1.1 \mathrm{~mm}$ and $\pm 1.2 \mathrm{~mm}$ respectively, which were slightly better than $5 \mathrm{~mm}$ tolerance values of $\pm 1 \mathrm{~mm}$. Fig. 6 provides the summary of results, it is clear that $4 \mathrm{~mm}$ and $5 \mathrm{~mm}$ ball lens gives optimized results providing high Q-factor and good tolerance values at BER of $10^{-12}$. These experimental tolerance ranges were in good agreement with the simulation results obtained in [8].

\section{Ball Lens Cap Design for Photodiode Custom Packaging}

Based on the laboratory test-bench results, we realized an OWC system prototype including $4 \mathrm{~mm}$ ball lens. We choose this diameter lens because of its good signal quality at the receiver and its convenience to package it on top of the TO-46 header of the photodiode (which has a diameter of $4.7 \mathrm{~mm}$ ). We designed the can cap for a $4 \mathrm{~mm}$ ball lens based on results provided by the ray-tracing simulations. The critical step was to place photodiode active area at the focal point of the ball lens, 


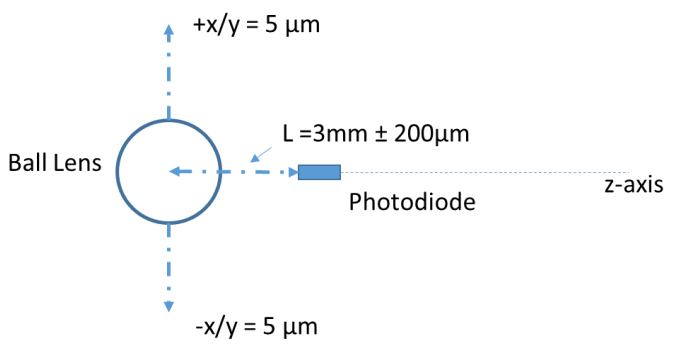

Figure 7: Ball lens position deviation in radial and axial direction w.r.t photodiode active area.

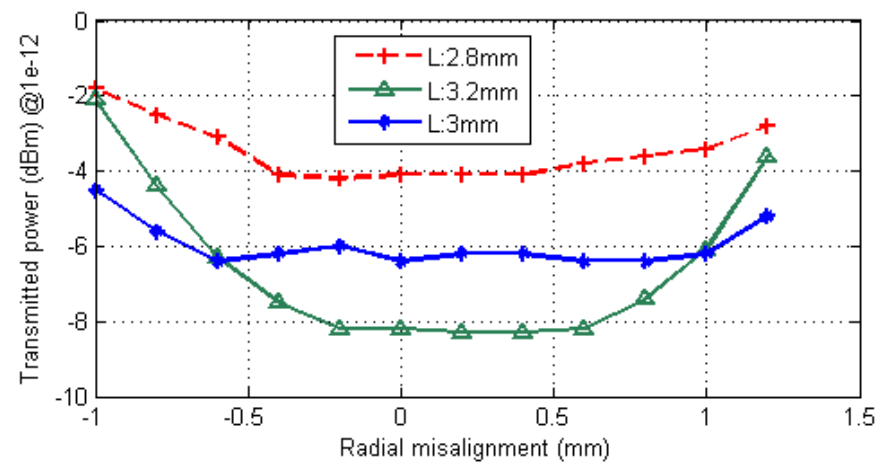

Figure 8: Transmitted power as a function of radial misalignment considering $5 \mu \mathrm{m}$ deviation on $\mathrm{x}-\mathrm{y}$ axis and for different values of $\mathrm{L}$ (z-axis).

considering the precision of the packaging. The precision given by the packaging lab was $\pm 5 \mu \mathrm{m}$ on $\mathrm{X}$-Y axis, and $\pm 200 \mu \mathrm{m}$ on Z-axis. Thus, we performed again a simulation of system misalignment at $10 \mathrm{~cm}$ distance, taking into account a radial (X/Y axis) and an axial (Z-axis, $\mathrm{L})$ deviation of the ball lens position, respect to optimal position. On X/Y-axis, we considered a $5 \mu \mathrm{m}$ deviation. On z-axis, we considered a deviation of $\pm 200 \mu \mathrm{m}$ respect to a reference value of $L=3 \mathrm{~mm}$ (optimal position, see Fig.7).

The designed OWC system is limited by the VCSEL transmitted power of $1 \mathrm{~mW}(0 \mathrm{dBm})$. It was observed that, for a radial deviation of $5 \mu \mathrm{m}$, the tolerance range of approximately $\pm 1 \mathrm{~mm}$ can be obtained with $\pm 200 \mu \mathrm{m}$ deviation on z-axis (all the curves are below the $0 \mathrm{dBm}$ transmitted power).
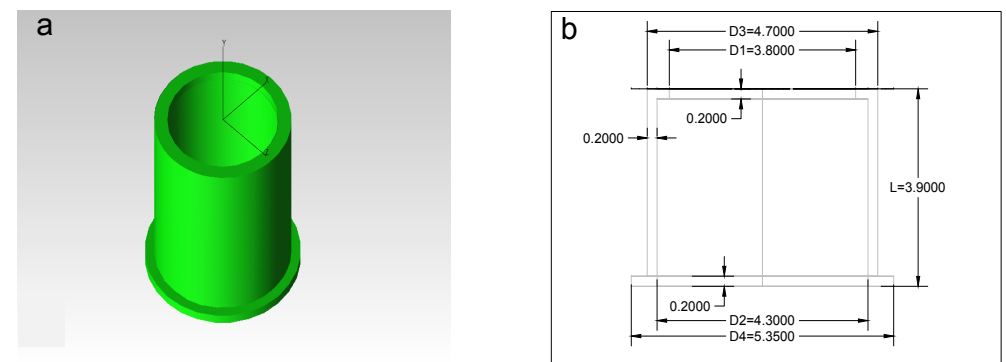

Figure 9: (a) Ball Lens can cap 3D view; (b) Can cap dimension. 


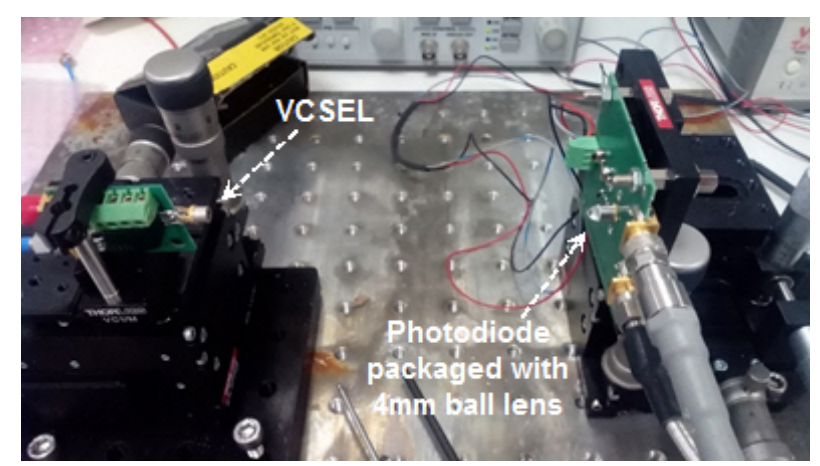

Figure 10: Experimental Setup of $2.5 \mathrm{~Gb} / \mathrm{s}$ at $10 \mathrm{~cm}$ transmission distance using VCSEL and custom designed photodiode packaging

The ball lens holder was designed according to TO-46 cap standards. Figure 9(a and b), shows the $3 \mathrm{D}$ view and the detailed dimensions of the designed cap. The final prototype and setup is presented in figure 10 .

\section{Prototype test and results}

The OWC system prototype was tested using the same setup shown in Fig. 2. Both the VCSEL and photodiode packaged with $4 \mathrm{~mm}$ ball lens were placed on a 3-axis stage at distance of $10 \mathrm{~cm}$ from each other. As previously discussed, we properly aligned the transmitter and the receiver by maximizing the Q-factor value.

Figure 11(a) reports the BERs as a function of the radial misalignment, illustrating the tolerance range of the OWC system prototype. We observed a tolerance range of $\pm 1.1 \mathrm{~mm}$, matching with the results of the test-bench experiment, reported in section 2 . Wide eye diagram for $2.5 \mathrm{~Gb} / \mathrm{s}$ is shown in Fig. 11(b) for the aligned system position $(x=y=0)$. These results highlight the successful implementation of the $2.5 \mathrm{~Gb} / \mathrm{s}$ transmission system prototype at $10 \mathrm{~cm}$ of distance with the tolerance range of $\pm 1 \mathrm{~mm}$ at BER of $10^{-12}$.
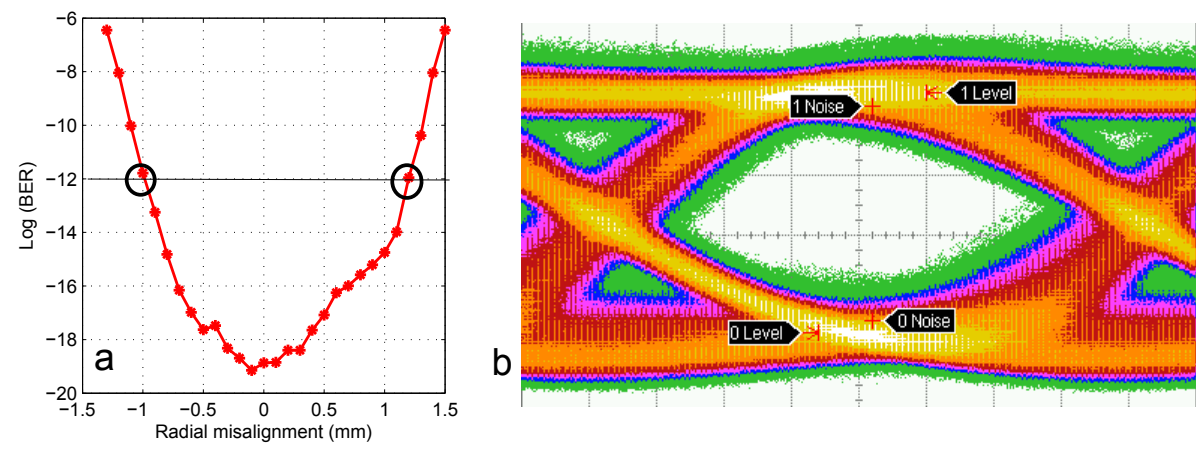

Figure 11: (a) BER as a function of radial misalignment for system prototype; (b) Eye diagram of $2.5 \mathrm{~Gb} / \mathrm{s}$ transmission using system prototype. 


\section{Conclusion}

In this paper, we demonstrated an OWC system test-bench providing $2.5 \mathrm{~Gb} / \mathrm{s}$ at $10 \mathrm{~cm}$ distance with a passive misalignment tolerance of $\pm 1 \mathrm{~mm}$ considering a target BER of $10^{-12}$. The system was based on a VCSEL as transmitter and on an InGaAs photodiode with ball lens as receiver and it was made to be suitable for the HEP particle detectors. The VCSEL was chosen because of its radiation hardness and the selected wavelength $(1550 \mathrm{~nm})$ was outside the absorption spectrum of the silicon strip sensors. The InGaAs photodiode with small area was already demonstrated to be radiation hard [9], while the fused silica/quartz ball lens has to be tested to proof the reliability in particle detectors. We already planned future experiments to test all the devices in high dose X-rays environment. Based on the test-bench results, we realized a system prototype with a packaged receiver. Finally, we tested this prototype with $2.5 \mathrm{~Gb} / \mathrm{s}$ transmission at $10 \mathrm{~cm}$ of distance. The tolerance to misalignment in this case was of $\pm 1 \mathrm{~mm}$, matching the test-bench result. This tolerance range satisfies the requirements for establishing the wireless link between the layers of silicon detector modules in the CMS inner tracker.

\section{Acknowledgment}

The research leading to these results has received funding from the People Programme (Marie Curie Actions) of the European Unions Seventh Framework Programme FP7/2007-2013/ under REA grant agreement n [317446] INFIERI "INtelligent Fast Interconnected and Efficient Devices for Frontier Exploitation in Research and Industry". Authors would also like to thank Dr. Giovan Battista Preve and Dr. Marco Chiesa from TeCIP packaging labs for extending their support in photodiode packaging.

\section{References}

[1] CMS collaboration, The CMS tracker system project: Technical design report, CERN-LHCC-98-006

[2] J. Troska et al., Optical Readout and Control Systems for the CMS Tracker, IEEE Trans. Nucl. Sci 50 (2003) 233.

[3] J. Chramowicz et al., Free-space optical interconnects for cable-less readout in particle physics detectors, JINST 52010C12038.

[4] D. Underwood, et al., optical technology for low mass intelligent trigger and readout, 2010 JINST $5 \mathrm{C} 07011$.

[5] S. S. E. Nasr-Storey, et al., Effect of radiation on a mach-zehnder interferometer silicon modulatorfor HL-LHC data transmission applications, IEEE Trans. Nucl. Sci 62 (2015) 329-335.

[6] E.Bisaillon et al., Free-space optical link with spatial redundancy for misalignment tolerance, 2002 IEEE Photon. Techol Lett. 14.

[7] Ke Wang et al., High-speed free-space based reconfigurable card-to-card optical interconnects with broadcast capability, 2013 Optics express. 21.

[8] W.Ali. et al., Optical Wireless Communication system for particle detectors in high energy physics, 2015 Nucl. Instr and Methods in Physics research A, 824. 
[9] J. Troska, et al., Radiation damage studies of lasers and photodiodes for use in multi-gb/s optical data links, 2011 IEEE Trans. Nucl. Sci 58.

[10] S. J. Akhtar, et al., A study of neutron and gamma radiation effects on transmission of various types of glasses, optical coatings, cemented optics and fiber, 2007 Optical Materials 2912.

[11] G. P Agrawal,. Fiber-Optic Communication Systems, New York: Wiley-Interscience, 2002. Print. 